\title{
Multiprocessor Scheduling of a Multi-mode Dataflow Graph Considering Mode Transition Delay
}

\author{
HANWOONG JUNG, Seoul National University \\ HYUNOK OH, Hanyang University \\ SOONHOI HA, Seoul National University
}

\begin{abstract}
Synchronous Data Flow (SDF) model is widely used for specifying signal processing or streaming applications. Since modern embedded applications become more complex with dynamic behavior changes at runtime, several extensions of the SDF model have been proposed to specify the dynamic behavior changes while preserving static analyzability of the SDF model. They assume that an application has a finite number of behaviors (or modes) and each behavior (mode) is represented by an SDF graph. They are classified as multi-mode dataflow models in this paper. While there exist several scheduling techniques for multi-mode dataflow models, no one allows task migration between modes. By observing that the resource requirement can be additionally reduced if task migration is allowed, we propose a multiprocessor scheduling technique of a multi-mode dataflow graph considering task migration between modes. Based on a genetic algorithm, the proposed technique schedules all SDF graphs in all modes simultaneously to minimize the resource requirement. To satisfy the throughput constraint, the proposed technique calculates the actual throughput requirement of each mode and the output buffer size for tolerating throughput jitter. We compare the proposed technique with a method which analyzes SDF graphs in each execution mode separately and a method that does not allow task migration for synthetic examples and three real applications: H.264 decoder, vocoder, and LTE receiver algorithms.
\end{abstract}

CCS Concepts: $\bullet$ Theory of computation $\rightarrow$ Streaming models; •Computer systems organization $\rightarrow$ Embedded software;

Additional Key Words and Phrases: Synchronous dataflow, Multi-mode dataflow, Mode transition delay, Task migration, Throughput requirement

ACM Reference Format:

Hanwoong Jung, Hyunok Oh, and Soonhoi Ha, 2016. Multiprocessor Scheduling of a Multi-mode Dataflow Graph Considering Mode Transition Delay. ACM Trans. Embedd. Comput. Syst. 9, 4, Article 39 (March 2010), 21 pages.

DOI : 0000001.0000001

\section{INTRODUCTION}

Model-based design methodology is widely accepted for embedded system design since it enables us to cope with ever increasing system complexity by maximizing the benefit of abstraction. As an algorithm specification model, this paper adopts a coarse-grain dataflow model which is suitable for specifying signal processing or streaming applications. In a dataflow graph, a node presents a function module and an arc represents

This research was supported by a grant to Bio-Mimetic Robot Research Center Funded by Defense Acquisition Program Administration, and by Agency for Defense Development (UD130070ID), Basic Science Research Program through the National Research Foundation of Korea (NRF) funded by the Ministry of Science, ICT \& Future Planning (NRF-2013R1A2A2A01067907, 2013R1A1A1013384), and IT R\&D program MKE/KEIT (No. 10041608, Embedded system Software for New-memory based Smart Device).

Author's addresses: H. Jung and S. Ha, Department of Computer Science and Engineering, Seoul National University; H. Oh, Department of Information System, Hanyang University;

Permission to make digital or hard copies of all or part of this work for personal or classroom use is granted without fee provided that copies are not made or distributed for profit or commercial advantage and that copies bear this notice and the full citation on the first page. Copyrights for components of this work owned by others than ACM must be honored. Abstracting with credit is permitted. To copy otherwise, or republish, to post on servers or to redistribute to lists, requires prior specific permission and/or a fee. Request permissions from permissions@acm.org.

(C) 2010 ACM. 1539-9087/2010/03-ART39 $\$ 15.00$

DOI : 0000001.0000001 
the flow of data samples (or tokens) through the FIFO channel between two end nodes. When a node is invoked, it consumes a specified number of data samples (called sample rate) from each input arc and produces a specified number of samples to each output arc. A node becomes executable when all input arcs have as many data samples as the specified sample rates. If the sample rate is a fixed integer number which does not change at run-time, the dataflow graph is called a synchronous dataflow (SDF) graph [Lee and Messerschmitt 1987].

For a given multiprocessor system, we need to determine the mapping of nodes to the processors and the execution order of mapped nodes on each processor. The static sample rate in the SDF model allows us to make the mapping and scheduling decision statically. From the static mapping and scheduling result for an SDF graph on a multiprocessor, we can estimate the performance and the resource requirement, which is very desirable for the design of embedded systems with tight real-time and resource constraints. If the implemented system follows the pre-determined mapping and execution order of nodes at run-time, the system can be claimed to be "correct by construction".

But the SDF model has a severe restriction to be used for modern embedded applications. It cannot express the dynamic behavior of an application, while modern embedded applications become more complex with dynamic behavior changes at runtime. For example, advanced video CODEC algorithms have several function modules that are conditionally invoked depending on the contents of the input frame. In addition, an application may have multiple implementations of the same algorithm to support various levels of quality of service.

To express such dynamic behavior of an application in the SDF model with keeping the static analysis capability, several extensions have been proposed to the SDF model, including FSM-based scenario-aware dataflow (FSM-SADF) [Stuijk et al. 2011], parameterized SDF (PSDF) [Bhattacharya and Bhattacharyya 2001], MTMSADF [Jung et al. 2014], mode-aware dataflow (MADF) [Zhai 2015], and so on [Girault et al. 1999] [Wiggers et al. 2008]. They all assume that an application has a finite number of behaviors (or modes) and each behavior (mode) can be represented by an SDF graph. We denote those MoCs (Models of Computation) as multi-mode dataflow (MMDF) graphs in this paper and define a representative MMDF model that can be implemented by any specific extension.

Because an MMDF graph is composed of SDF graphs in multiple modes, the static schedule of the SDF graph on each mode can be constructed to estimate the overall performance and the resource requirement of the MMDF graph. Also, for more accurate estimation, the mode transition delay during mode changes should be considered. If the mode change occurs frequently and periodically, the mode transition delay will seriously degrade the overall performance of the MMDF graph. While there exist several techniques to schedule a multi-mode dataflow graph with considering the mode transition delay, the existing approaches do not allow task migration between modes. However, we observe that the resource requirement for a given throughput constraint can be additionally reduced if task migration is allowed. Since task migration will cause additional run-time overhead during mode transitions, we should take into account the effect task migration overhead on the throughput performance.

In this paper, we propose a multiprocessor scheduling technique of a multi-mode dataflow graph considering the mode transition delay conservatively. Our scheduling objective is to minimize the number of processors allowing task migration among all modes, while satisfying the overall throughput constraint. Also, from the scheduling result, we compute the output buffer size to tolerate the time fluctuation of output results. Experiment results show that the proposed scheduling approach provides better solutions than the existing approaches which determine the mapping and the 
scheduling for graphs either each mode independently incurring task migration between modes, or all modes at the same time disallowing task migration.

The rest of this paper is organized as follows. The next section gives a motivational example to clarify the problem addressed in this paper, and introduces the key idea of the proposed technique. Section 3 reviews the related work. The problem addressed in this paper will be defined and formulated in Section 4. In Section 5 and 6, the throughput requirement analysis technique and the proposed scheduling technique considering the mode transition delay are explained in detail, respectively. In Section 7. we discuss our experimental results, and draw conclusions in Section 8 .

\section{MOTIVATIONAL EXAMPLE}

\subsection{Throughput Requirement Calculation Considering Mode Transition Delay}

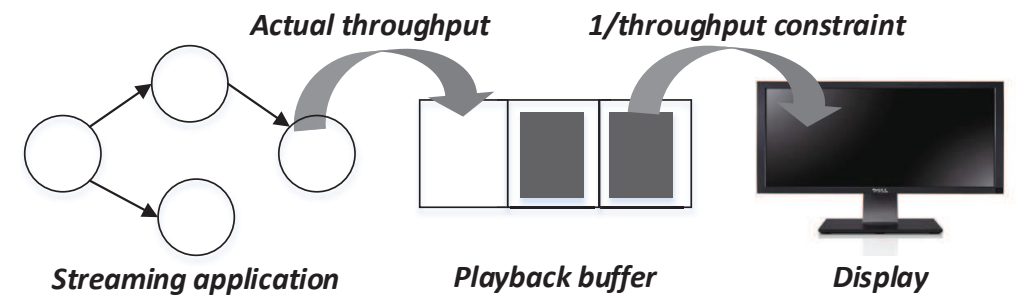

(a) Relation between the playback buffer and throughput

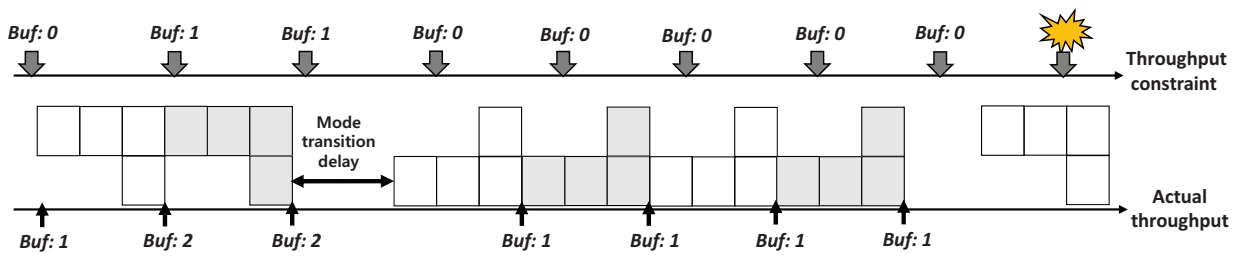

$<$ Timeline of schedule $1>$

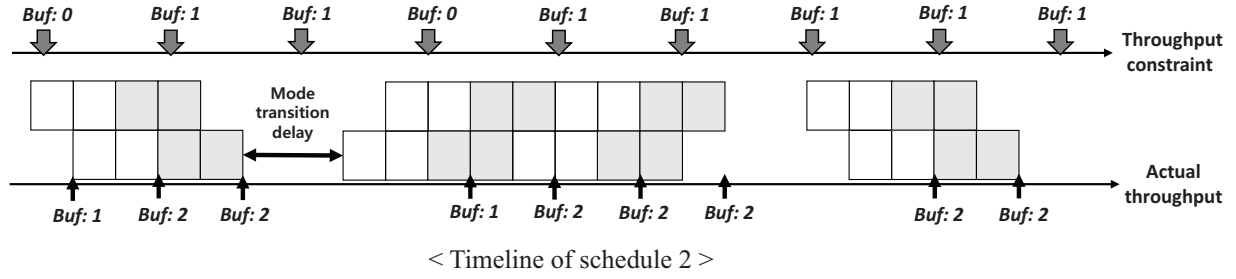

(b) Throughput requirement considering the mode transition delay

Fig. 1. Motivational example of throughput requirement calculation considering the mode transition delay

To cope with fluctuation of output intervals of streaming applications, an output buffer is usually used to obtain the steady output stream in streaming applications as depicted in Figure 1(a). The system will require data from the output buffer periodically and the period is defined by the inverse of the throughput constraint. If the throughput performance of an application is lower than the throughput constraint, the output buffer will be eventually empty. 
If a streaming application is specified in an MMDF graph, it can be scheduled at compile-time to meet the given throughput constraint. To guarantee the throughput constraint, not only throughput performance of each mode, but also the mode transition delay should be considered. Although a mapping/scheduling result of each SDF graph keeps the throughput constraint, the average throughput performance can be lower than the constraint because of additional time delay during mode transition.

Various factors have an effect on the mode transition delay. In [Stuijk et al. 2010] and [Geilen et al. 2012], the system reconfiguration overhead and DVFS delay are considered as the mode transition delay. Also, [Zhai 2015] defines the mode transition delay to quantify the proposed transition protocols. Especially, it proposes a MOO (Maximum-Overlap Offset) transition protocol which calculates an offset that guarantees no interference between the execution of both old and new modes. When a mode transition occurs, an application will be delayed until this offset, which should be considered in the throughput calculation. In addition to such rescheduling delay, we need to consider the task migration overhead in the computation of mode transition delay.

Since the mode transition delay degrades the average throughput of an MMDF application, the throughput constraint can be violated even if the throughput of each mode is higher than the throughput constraint. Figure 1(b) shows an example of two different schedules for an MMDF graph. The MMDF graph consists of two different modes, and there exists additional time delay during mode transition. As represented by arrows on an upper line, the system dequeues data from the output buffer periodically with the same rate as the throughput constraint. The arrows on a lower line tell when an MMDF application enqueues data to the output buffer. A number annotated on each arrow denotes the number of data in the output buffer after the access is completed.

In case of schedule 1 in Figure 1(b), even though the schedule of each mode satisfies the throughput constraints, the throughput constraint is eventually violated since the mode transition delay is accumulated. To avoid this problem, we need to set the throughput constraint of each mode tighter than that of the application as schedule 2 illustrates in the figure; it keeps the throughput constraint because it fills the output buffer faster than the throughput constraint. Therefore we need to calculate the actual throughput requirement for each mode considering the mode transition delay, in order not to violate the given constraint. Details will be discussed in Section 5 .

\subsection{Task Migration Between Mode Transition}

Figure2 (a) shows an MMDF graph example which consists of two modes: $M 1$ and $M 2$. We assume that the throughput constraint of the MMDF graph is given as 1/35. Each execution mode of an MMDF graph is represented with an SDF graph. The execution time and sample rates of each node may vary depending on the execution mode. In mode $M 1$, the execution times of nodes $A, B, C$, and $D$ are $17,13,14$, and 16 , respectively and in mode $M 2$, they are $12,10,8$, and 10 . The output sample rates of nodes $B$ and $C$ are unity in mode $M 1$ while they are 3 in mode $M 2$. Refer to Section 4 for the formal description of the MMDF model assumed in this paper. Also, in this section, we only consider the task migration overhead as the mode transition delay to simply show the effect of task migration during mode transition.

A naive approach to schedule an MMDF graph is to schedule an SDF graph in each mode independently with multiple objectives of resource minimization and throughput maximization. For example, for the given throughput constraint, we find an optimal mapping/scheduling result in each execution mode as shown in Figure 2 (b). Since it does not consider mapping results in the other modes, a node may be mapped onto different processors between modes. Therefore, the mapping result requires task mi- 


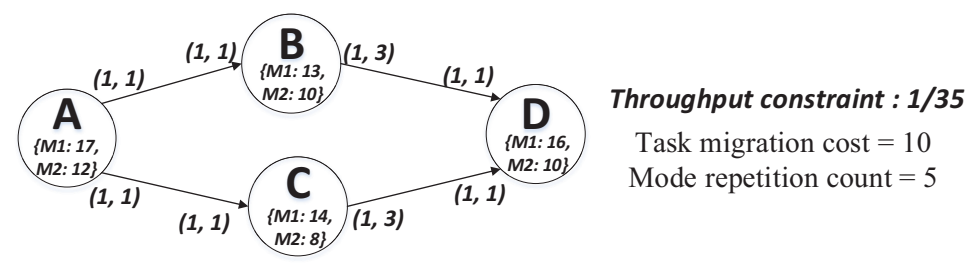

(a) An MMDF graph example
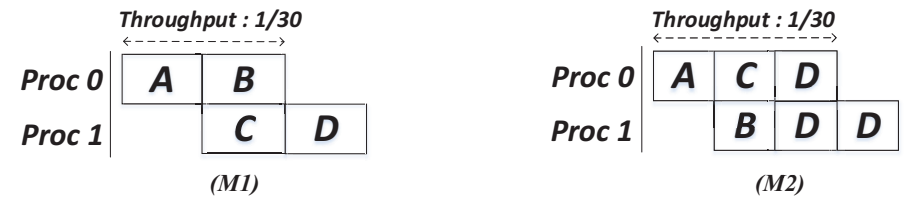

(b) Mapping/scheduling result when each SDF graph is scheduled independently

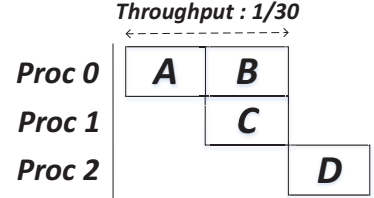

(M1)

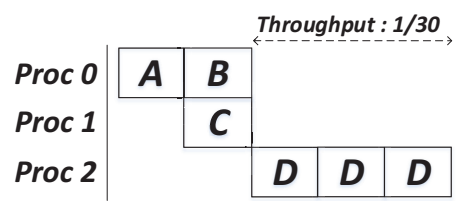

(M2)

(c) Mapping/scheduling result considering all modes simultaneously disallowing task migration
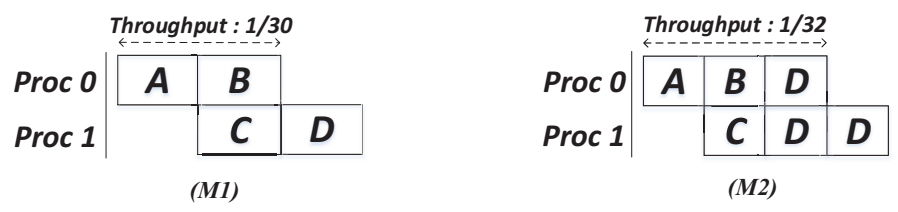

(d) Mapping/scheduling result considering all modes and task migration

Fig. 2. Motivational Example of Task Migration

gration when the mode changes. In Figure 2(b), nodes $B, C$ and $D$ will be migrated to other processors when the mode transition occurs.

Another approach to schedule an MMDF graph is to consider all modes simultaneously disallowing task migration [Stuijk et al. 2008] [Geilen and Stuijk 2010]. Since the mapping is constrained in these approaches, the scheduling results generally require more processors than those that allow task migration. For instance, three processors are required to meet the given throughput constraint for the mapping/scheduling result without task migration as shown in Figure 2 (c), while two processors are enough for the scheduling result with task migration in Figure 2(b). Since the objective of this paper is to minimize the resource requirement under a given throughput constraint, the proposed approach allows task migration. Their approach is used as a reference technique for comparison with the proposed technique in experiments. 
Consider the former approach that allows task migration in Figure 2 (b). If the mode transition occurs frequently and the task migration overhead is non-negligible, then the given throughput constraint may not be satisfied. For instance, assume that the mode transition occurs every 5 iterations and the task migration overhead of each node is 10. In Figure 2 (b), 30 time unit is added every 5 iterations because nodes $B, C$ and $D$ should be migrated for mode transition. Then, the output buffer will be eventually empty, because the average throughput performance of the MMDF graph becomes lower then the throughput constraint.

Therefore, in this paper, we propose another approach that schedules the SDF graphs of all modes simultaneously allowing task migration among execution modes. Figure 2 (d) shows a mapping and scheduling result produced by the proposed technique. It requires 2 processors and only 10 additional time units for task migration, which may satisfy the throughput requirement with proper output buffering. Throughput analysis considering task migration overhead will be discussed in Section 5 .

\section{RELATED WORK}

As the related work to the proposed technique, we review several extensions of the SDF model that have been proposed to express the dynamic behavior of an application.

One of the most representative multi-mode dataflow models is FSM-based SADF (Scenario-Aware Data Flow) model [Stuijk et al. 2008], shortly FSM-SADF. In the FSM-SADF model, an application consists of multiple scenarios (modes) and each scenario is specified by an SDF graph. To specify multiple scenarios and their transitions, it defines a special control task called detector that has an FSM inside. The detector task sends the control information to the normal computation tasks that may change its behavior. For the FSM-SADF model, several techniques to statically analyze the timing behavior such as worst case latency and throughput [Geilen and Stuijk 2010] have been proposed. Also, a binding-aware scenario graph [Stuijk et al. 2010] has been proposed to take into account the resource constraint. And, in [Damavandpeyma et al. 2013], it considers reconfiguration overhead for DVFS (Dynamic Voltage Frequency Scaling) as the mode transition delay. However, it only considers the worst-case performance analysis of the FSM-SADF graph for the given task mapping, and requires inherently exponential time-complexity for exact analysis.

As a similar model to the FSM-SADF, an MTM-SADF [Jung et al. 2014] has been proposed to specify application level dynamism based on an SDF. Instead of an FSM, it uses a Mode Transition Machine (MTM) which is a simplified form of the FSM to represent the mode transition. It proposes a hybrid task mapping technique with minimizing the overall energy consumption under the throughput constraints. However, it analyzes each SDF graph independently.

PSDF (Parameterized Synchronous Data Flow) [Bhattacharya and Bhattacharyya 2001] proposes a meta-modeling technique for run-time adaptation of parameters in a structured way. In the PSDF model, the dynamic behavior of a task is modeled by parameters and the task behavior can change at the iteration boundary at run-time. Since the PSDF becomes an SDF graph at each iteration, the PSDF can be regarded as a multi-mode dataflow graph that may change modes every iteration.

MCDF (Mode-Controlled Data Flow) [Moreira 2012] is one of data flow MoCs which enables the expression of the data-dependent functional behavior. However, it mainly focuses on SDR (Software-Defined Radio) applications, where different sub-graphs need to be active in different modes.

In VRDF (Variable-Rate Data Flow) [Wiggers et al. 2008] model, it allows variable port rates within a specified range, and VPDF (Variable-rate Phase Data Flow) [Wiggers et al. 2008] is proposed to combine characteristics of VRDF and CSDF where each actor has a sequence of phases, and for every phase, the number of firings can be 
parameterized. For these MoCs, buffer size analysis technique is proposed to satisfy a throughput constraint.

MADF (Mode-Aware Data Flow) [Zhai 2015] has been proposed to support hard real-time scheduling for multi-mode CSDF (Cyclo-Static Data Flow) model [Bilsen et al. 1995]. It combines advantages of SADF and VPDF to specify application level dynamism. Also, it proposes MOO (Maximum-Overlap Offset) mode transition protocol to derive an efficient analysis for a hard real-time scheduling of an MADF graph. With this mode transition protocol, the timing behavior of individual modes and during mode transitions can be analyzed independently.

BPDF (Boolean Parametric Data Flow) [Bebelis et al. 2013] supports change of port rates and graph topology at run-time using integer and boolean parameters. In BPDF model, integer parameters are used to change port rates at each iteration, and boolean parameters are used for activation and deactivation of edges to change graph topology.

HDF (Heterogeneous Data Flow) (or *-chart) [Girault et al. 1999] supports multimode applications through an FSM that executes an iteration of an SDF graph in each state. So, an application is specified with a set of different SDF graphs combined with an FSM.

While various analysis and scheduling techniques have been proposed for those MoCs, no one considers task migration between modes. In [Lee et al. 2013], task migration is considered in the failure-aware task scheduling technique where an SDF graph is scheduled multiple times with different number of processors allocated, aiming to maximize the throughput with the allocated number of processors. When a processor fails in the middle of execution, it changes the schedule that uses the reduced number of processors by one. Then task migration occurs between two different schedules before and after processor failure. They try to minimize the migration cost between two SDF schedules. This method is similar to the base method that will be used for comparison in this paper: schedule each mode separately and find the best processor-to-processor mapping (or processor renaming) in order to minimize the migration cost.

In summary, to the best of our knowledge, this paper is the first work which proposes a multiprocessor scheduling technique of an MMDF graph allowing task migration between modes, and analyzes the throughput requirement considering the mode transition delay.

\section{PROBLEM DEFINITION}

The MMDF model assumed in this paper is not a specific model but a generic model encompassing existing similar models such as FSM-SADF [Stuijk et al. 2008] and MTMSADF [Jung et al. 2014]. In those models, mode transition is specified by an FSM and all modes are integrated into a single graph with varying configuration parameters. Figure 3 shows an MMDF graph example. We first define the MMDF model and the problem formally.

Application model: An MMDF graph is specified by a combination of a task graph and a mode transition graph $(M T G)$, or $(T, C, D) \times M T G$, where

- MTG is specified by a tuple (Mode, Trans) where Mode is a finite set of modes and Trans is a finite set of transitions. Trans is specified as follows: Trans = $\{(\mathrm{cm}, \mathrm{nm}) \mid \mathrm{cm} \in$ Mode, $\mathrm{nm} \in M o d e\}$ where $\mathrm{cm}$ denotes a current mode and $\mathrm{nm}$ denotes a next mode.

- $T$ is a finite set of computational tasks. Each task $t \in T$ has a set of ports $P_{t}$ to send/receive data to/from other adjacent tasks. $P_{t}=I P_{t} \cup O P_{t}$ where $I P_{t}$ is a set of input ports and $O P_{t}$ is a set of output ports. For each port $p \in P_{t}$, it is assigned a fixed 


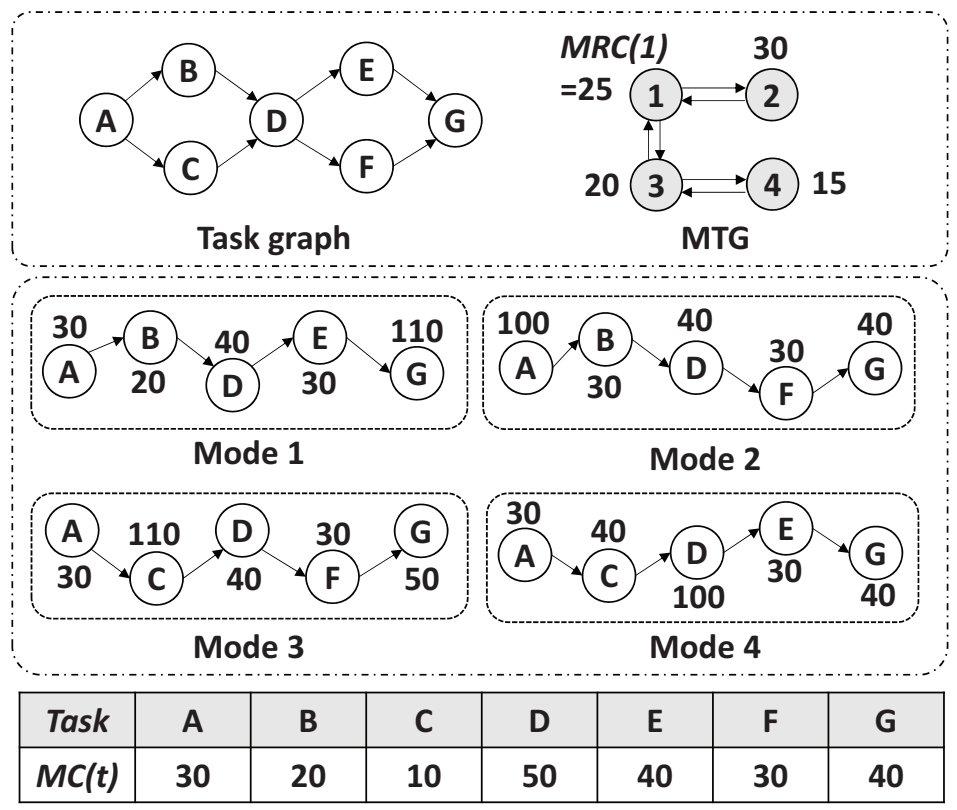

Fig. 3. An MMDF graph example

rate, Rate ( $p$, mode), in each execution mode. Thus the graph becomes an SDF graph for each mode.

$-C$ is a finite set of FIFO channels. A channel defines a one-to-one connection between two end ports.

$-D$ is a set of the number of initial tokens in all channels. $d_{c}(m) \in\{0\} \cup \mathbb{N}$ for $\forall d_{c} \in D$ is the number of initially stored tokens in the channel $c$ in mode $m$.

Architecture model: the target architecture consists of a set of processing elements.

$-P E$ is a set of processing elements. For each $p \in P E$ and $m \in \operatorname{Mode}, \operatorname{Map}(m, p)=$ $\{t \mid t \in T$ where $t$ is mapped onto a processor $p$ in mode $m\}$

Note that even though the proposed technique is applicable to heterogeneous multiprocessor systems, this paper assumes a homogeneous multiprocessor system for simple explanation and implementation.

To analyze the scheduling performance of an MMDF graph, we assume profiling information is available as follows:

\section{Profiling information}

- Worst case execution time (WCET) for each task $t \in T$ and $m \in$ Mode is given as $W C E T\left(t_{m}, p\right)$ for each processing element $p \in P E$ of the target architecture. In Figure 3, the WCET of a node is annotated in each mode. For example, WCET of node $A$ is 30 in modes 1,3 , and 4 , and 100 in mode 2 .

- For each $m \in$ Mode, we are given a minimum number of iterations that the application stays at the mode, which is denoted by $M R C(m)$ where $M R C$ stands for the minimum repetition count. As $M R C$ becomes smaller, the mode transition occurs 
more frequently. A mode is associated with an $M R C$ value as shown in Figure 3 where $M R C$ is $25,30,20$ and 15 in modes 1, 2, 3, and 4, respectively.

- For each $t \in T$, task migration cost is given by $M C(t)$. If the system is a distributed memory system, the migration cost will include the time overhead of moving the code and the context of a task between two processors. If it is a shared memory system, the migration cost will be small as cold miss penalty for task execution. A table in Figure 3 shows the $M C$ value of each task. For instance, $M C$ of node $A$ is 30 .

We assume that the mode transition of an MMDF graph occurs at the iteration boundary of the SDF schedule associated with the current mode $(\mathrm{cm})$. For each task $t \in T$, after the mode transition to the next mode $(\mathrm{nm}), \operatorname{Rate}(\mathrm{p}, \mathrm{cm})$ of all $P_{t}$ and $W C E T\left(t_{c m}\right)$ changes to Rate $(p, n m)$ and $W C E T\left(t_{n m}\right)$. And the SDF schedule associated with the next mode is followed.

With those application and architecture models and profiling information, the problem addressed in this paper is summarized as follows:

PROBLEM: Find a mapping and scheduling result of an MMDF graph which satisfies the given throughput constraint

minimize. the number of required processors

subject to. the overall throughput performance of the MMDF graph should be higher than the given throughput constraint.

The proposed MMDF scheduling framework is based on a genetic algorithm. So, it needs to evaluate all candidate solutions in every iterations. How to evaluate whether a given mapping and scheduling result of an MMDF graph satisfies the given throughput constraint will be explained in the next section.

\section{THROUGHPUT REQUIREMENT ANALYSIS}

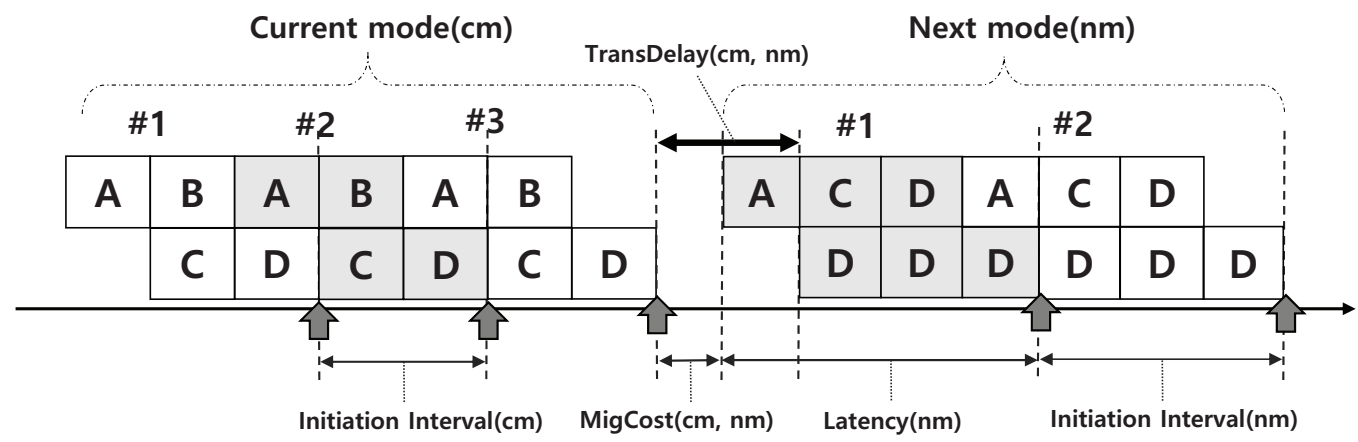

Fig. 4. Mode transition delay

In this section, we explain how to compute the throughput of an MMDF graph considering the mode transition delay with a given static scheduling results of modes. For simple coordination of task migration and conservative estimation of the mode transition overhead, we assume that mode transition and task migration is performed in a blocking fashion. In the blocking scheme, the task schedule is blocked at the mode transition boundary. Task migration is initiated after all tasks in the current mode finish and the next mode starts after task migration is completed. During mode transitions, 
no task is executed. Note that the throughput may be degraded due to the blocking even if there is no task migration when a mode transition occurs.

Figure 4 shows a mode transition scenario. It is assumed that a mode changes after the end of iteration 3 in the current mode $\mathrm{cm}$. Task $A$ at iteration 1 in next mode $\mathrm{nm}$ can start after task $D$ is completed at iteration 3 in mode $\mathrm{cm}$ and task migration is completed although a processor is available to execute task $A$ earlier. In the blocking scheme of task migration, the mode transition delay consists of two terms: task migration delay and block scheduling effect.

Definition 5.1 (Mode Transition Delay).

$$
\begin{array}{r}
\forall(\mathrm{cm}, \mathrm{nm}) \in \text { Trans, TransDelay }(\mathrm{cm}, \mathrm{nm})=\operatorname{Mig} \operatorname{Cost}(\mathrm{cm}, \mathrm{nm})+\operatorname{Latency}(\mathrm{nm}) \\
-\operatorname{InitiationInterval}(\mathrm{nm})
\end{array}
$$

where $\operatorname{Mig} \operatorname{Cost}(\mathrm{cm}, \mathrm{nm})$ represents the task migration delay between current mode $\mathrm{cm}$ and next mode $\mathrm{nm}$, Latency $(\mathrm{nm})$ denotes the latency between the earliest start time of a task and the latest finish time of a task in mode $\mathrm{nm}$, and InitiationInterval $(\mathrm{nm})$ is the start time interval between two consecutive iterations in mode $\mathrm{nm}$. Note that InitiationInterval $(\mathrm{nm})$ is equal to the time interval between two consecutive output samples in a streaming application without mode transition. The inverse of the initiation interval denotes the throughput performance if no mode transition occurs.

\subsection{Buffer Size Determination}

As discussed in Section 2.1, an output buffer is adopted to produce data samples periodically. Since the mode transition delay causes the jitter of output production in an MMDF application, the output buffer should be large enough to provide the data samples during mode transitions. The required output buffer size depends on the maximum mode transition delay and the throughput difference between the input stream and the output stream in the buffer.

To determine the buffer size, we compute the arrival curves of the input and the output streams in the buffer. The arrival curve of a stream informs the number of arriving (or departing) samples (y-axis) within a time interval (x-axis) as shown in Figure 5 [Thiele et al. 2000]. For conservative estimation, we utilize the maximum arrival curve for the output stream and the minimum arrival curve for the input stream.

In Figure 1(a), task Display dequeues data from the output buffer periodically with satisfying the throughput constraint, which is depicted as the output curve (gray solid line) in Figure 5. The black solid line represents the minimum arrival curve of the input stream which presents the number of generated samples to the output buffer. The buffer size is computed based on the minimum repetition count $(M R C)$, the inverse of the throughput, and the maximum mode transition delay among all possible transition scenarios to the mode. In each mode, we compute the buffer size and then choose the maximum buffer size in all modes.

The maximum mode transition delay to mode $m$ is computed as following:

Definition 5.2 (Worst-case Mode Transition Delay to mode m).

$$
\operatorname{MaxTransDelay}(m)=\max _{\substack{\forall(c m, n m) \\ \in T r a n s \\ n m=m}} \operatorname{TransDelay}(\mathrm{cm}, \mathrm{nm})
$$

Note that since the slope of the curve depends on the mode transition delay, the mode repetition count, and the throughput performance of the MMDF schedule, the buffer size is determined after constructing an MMDF schedule meeting the throughput constraints in all modes. 


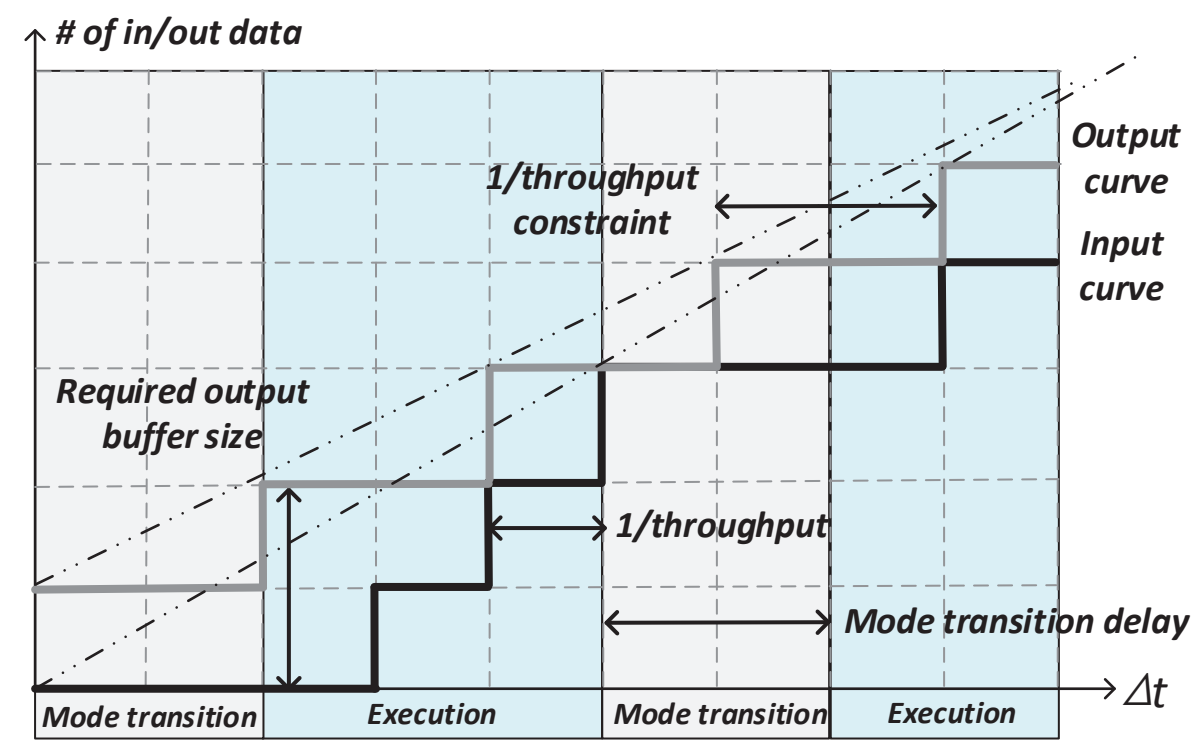

Fig. 5. Arrival curves for input and the output streams in the output buffer

From the arrival curves, we obtain the minimum output buffer size which is the maximum difference between the curves in every time interval $(\Delta t)$. If the overall throughput constraint is satisfied, the output buffer size is computed as following.

THEOREM 5.3 (OUTPUT BUfFER Size). The minimum size of the output buffer to satisfy the overall throughput constraint is decided by the following equation:

$$
\begin{gathered}
\text { Output buffer size }=\left\lceil\text { MaxInterval }_{\text {overall }} \times \text { ThrConst }\right\rceil \\
\text { where MaxInterval } \text { overall }=\max _{\substack{\forall(c m, n m) \\
\in T \text { Trans }}} \text { TransDelay }(\mathrm{cm}, \mathrm{nm})+\operatorname{InitiationInterval}(\mathrm{nm})
\end{gathered}
$$

PROOF. The buffer size is determined by the maximum distance between the input and the output curves, which is illustrated in Figure 5. It is evident that the maximum distance between two curves occurs during the first period of the input curve since the tangential slope of the input curve cannot be smaller than that of the output curve. Then the maximum distance is obtained just before the first jump of the input curve. Therefore,

$$
\begin{aligned}
\text { Output buffer size } & =\left\lceil\text { MaxInterval }_{\text {overall }} \div \frac{1}{\text { ThrConst }}\right\rceil \\
& =\left\lceil\text { MaxInterval }{ }_{\text {overall }} \times \text { ThrConst }\right\rceil
\end{aligned}
$$

\subsection{Throughput Requirement Analysis}

As discussed above, the throughput requirement at the next iteration depends on the mode transition delay and the minimum repetition counts of the mode. For conserva- 
tive estimation, the input curve should be steeper than the output curve in all modes. The throughput requirement in each mode can be formulated as follows:

Theorem 5.4 (Throughput REQUiREMENT). The throughput requirement in mode $m$ denoted as ThrRequire $(m)$, is formulated as following:

$$
\operatorname{ThrRequire}(m)=\frac{\text { ThrConst } \times M R C(m)}{M R C(m)-(\text { MaxTransDelay }(m) \times \text { ThrConst })}
$$

PROOF.

$$
\begin{gathered}
\text { The slope of input curve }=\frac{M R C(m)}{\text { MaxTransDelay }(m)+1 / \text { ThrRequire }(m) \times M R C(m)} \\
\text { And the slope of output curve }=\frac{1}{1 / \text { ThrConst }}=\text { ThrConst } \\
\text { Since the slope of input curve should be steeper than the output curve, } \\
\frac{M R C(m)}{\text { MaxTransDelay }(m)+1 / \text { ThrRequire }(m) \times M R C(m)} \geq \operatorname{ThrConst} \\
\text { and ThrRequire }(m) \geq \frac{M R C(m)}{M R C(m) / \text { ThrConst }-\operatorname{MaxTransDelay}(m)}
\end{gathered}
$$

If the throughput requirement calculated by Theorem 5.4 is not higher than the throughput in each mode for every MMDF graph, there is a task mapping/scheduling result which satisfies the throughput constraint considering the mode transition delay.

\section{PROPOSED MMDF SCHEDULING FRAMEWORK}

For MMDF scheduling problem, we adopt a genetic algorithm. The overall GA procedure of the proposed framework is shown in Figure 6

\subsection{GA Configuration}

Initialization \& Selection: Since a task (or node) can be mapped to different processors in modes, each task is regarded as a unit of mapping in each mode. The chromosome for GA is configured as shown in Figure 7, A chromosome is a set of mapping for each execution mode. Each gene of the chromosome represents to which processor a task in each execution mode is mapped. Chromosomes of initial population are randomly generated and selected for crossover and mutation. The number of selection is a configurable parameter of the GA framework.

Local optimization: In order to help the convergence of evolutionary process, a local optimization step is performed before the evaluation step. For local optimization, we devise a processor renaming heuristic that changes the processor id in each mode to reduce the migration cost. The details will be explained later.

Evaluation \& Replacement: In this step, we apply a list scheduling heuristic to find a static task schedule in each mode, based on the mapping information given by each chromosome. Once we construct a static schedule, we evaluate the fitness value of each offspring and check whether the throughput constraint is satisfied or not. The fitness function will be described in the next section. Chromosomes in the population are sorted by their fitness values and poor chromosomes are eliminated. 


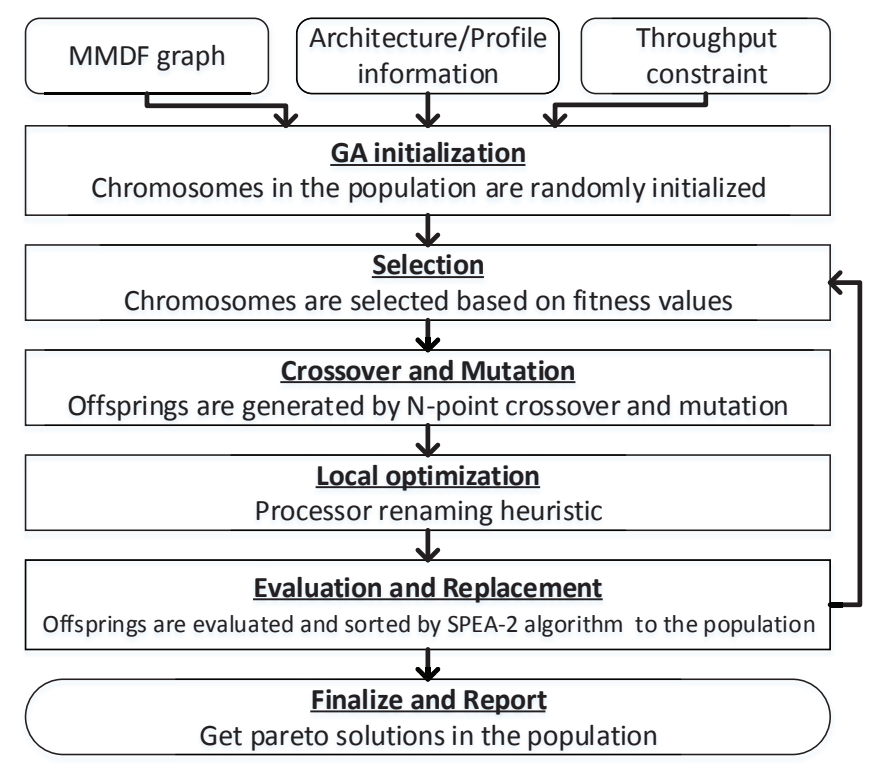

Fig. 6. The overall GA framework

Mapping for mode 0
\begin{tabular}{|l|l|l|l|l|l|l|l|l|}
\hline P0 & P1 & P2 & P1 & $\ldots$ & P1 & P2 & P2 & P0 \\
\hline
\end{tabular}

Fig. 7. Chromosome structure

\subsection{Fitness Function}

The objective of the MMDF scheduling is to minimize the number of processors. The required number of processors is defined as the maximum number of processors in all modes.

Definition 6.1 (The Number of Processors for an MMDF Graph).

$$
\begin{aligned}
& \text { The number of processor }=\max _{m \in \text { Mode }} \mid \text { Proc }_{m} \mid \\
& \text { where } \operatorname{Proc}_{m}=\{p \in P E \mid \operatorname{Map}(m, p) \neq \emptyset\}
\end{aligned}
$$

Since the large mode transition delay will degrade the throughput performance and more processors are likely to be required to meet the given throughput constraint, the mode transition delay including task migration overhead is considered to evaluate the number of required processors. And, the GA framework also aims to minimize the overall task migration cost as the secondary objective. The reduction of task migration will save energy consumption of the system, and reduce the network traffic in an NOC architecture. Therefore it is very desirable to reduce the total task migration cost (or delay) in an MMDF graph considering all mode transition scenarios; the total task migration cost of an MMDF graph is defined as follows: 
Definition 6.2 (Total Task Migration Cost).

$$
\begin{gathered}
\text { MigCost } \text { total }=\sum_{(\mathrm{cm}, n m) \in \operatorname{Trans}} \operatorname{Mig} \operatorname{Cost}(\mathrm{cm}, \mathrm{nm}) \\
\text { where MigCost }(\mathrm{cm}, \mathrm{nm})=\sum_{p \in P E} \sum_{\substack{t \in\{\operatorname{Map}(n m, p) \\
-\operatorname{Map}(\mathrm{cm}, p)\}}} M C(t)
\end{gathered}
$$

We sum up the migration cost of all possible migration scenarios that are defined by the $M T G$. For each transition in the $M T G$, we accumulate the migration cost of all tasks that are mapped to different processors after the mode transition.

\subsection{Local Optimization Technique}

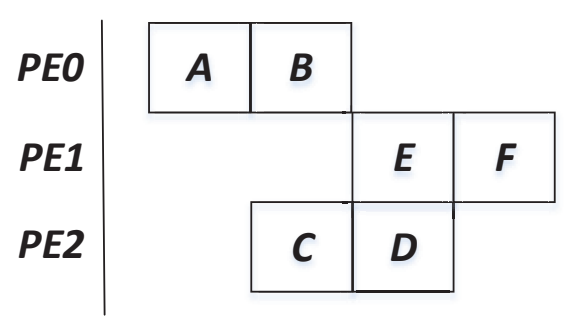

(a) Mapping in mode 0

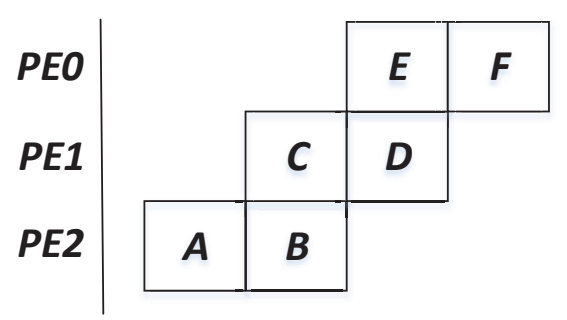

(b) Mapping in mode 1

Fig. 8. Without processor renaming, every task should be migrated when mode transition occurs. If $P E 0$ in mode 0 is renamed to $P E 2$ in mode $1, P E 1$ to $P E 0$, and $P E 2$ to $P E 1$, no task migration is required

Figure 8 shows a motivational example for local optimization, where two modes have different task mappings defined in the chromosome and a mode transition from mode 0 to mode 1 occurs. In the mapping result, all tasks should be migrated. However, since this paper assumes a homogeneous multiprocessor system, it is possible to rename the processor id in each mode, which is called processor renaming. If $P E 0$ in mode 0 is renamed to $P E 2$ in mode 1 then tasks $A$ and $B$ do not need to be migrated. Similarly, if $P E 1$ in mode 0 is renamed to $P E 0$ in mode 1 , and $P E 2$ to $P E 1$ then no task migration is required. Without the processor renaming technique, good solutions such as Figure 8 will be evaluated as poor solutions due to high migration delay, which seriously hinders the convergence of GA.

The time complexity of the processor renaming algorithm is given as $P^{M}$ where $P$ denotes the number of processors and $M$ is the number of mode transition scenarios. Therefore, we devise a simple greedy processor renaming heuristic as shown in Algorithm 1 to reduce the time complexity. In the proposed heuristic, the time complexity becomes $O\left(P^{2} \times M\right)$. Note that processor renaming is only applicable for homogeneous processor systems.

The heuristic measures the similarity between processors. The similarity between processors is defined by how many tasks are mapped on both processors in common.

Definition 6.3 (Similarity between processors).

$$
\text { For }(\mathrm{cm}, \mathrm{nm}) \in \text { Trans, Similarity }\left(p_{i}, p_{j}\right)=\left|\operatorname{Map}\left(\mathrm{cm}, p_{i}\right) \cap \operatorname{Map}\left(n m, p_{j}\right)\right|
$$

For each mode transition, processors in the next mode are renamed to the processors in the current mode with the maximum similarity. Even though the proposed heuristic does not consider all possible processor renaming scenarios and does not provide the 


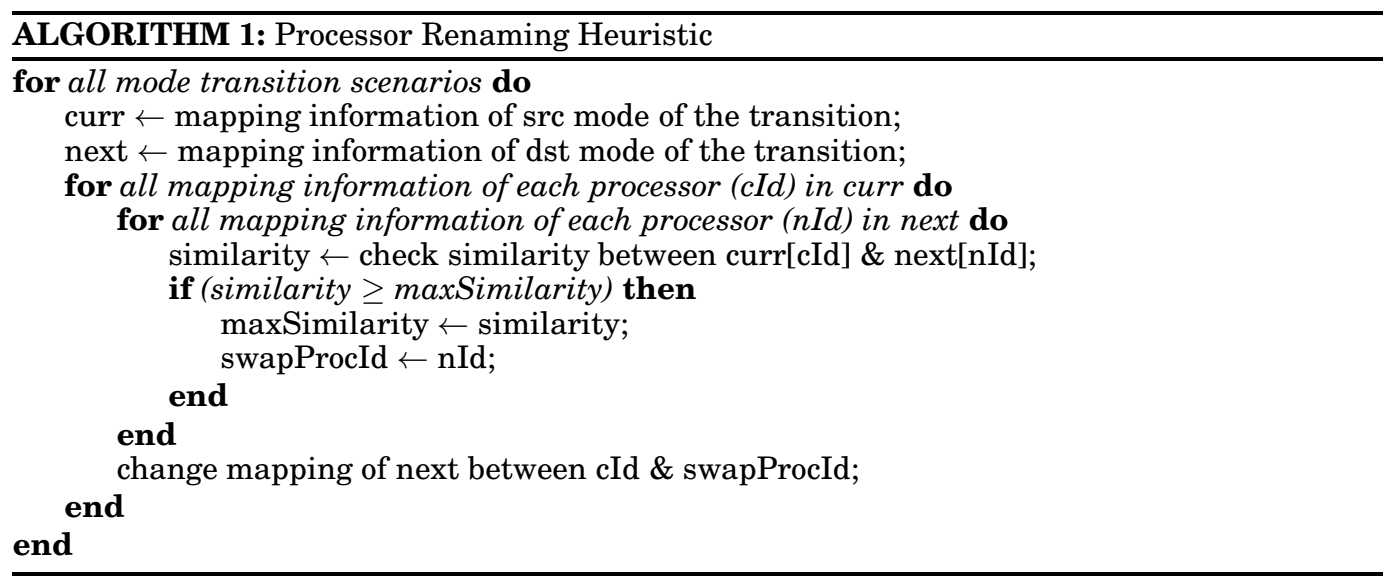

optimal renaming result, it reduces the time complexity significantly while generating good quality solutions as confirmed by experimental results.

\section{EXPERIMENTAL RESULTS}

To prove the viability of the proposed framework, we experiment with four synthetic examples and three real applications: H.264 decoder, vocoder [Zhai 2015] and LTE receiver [Siyoum et al. 2011] algorithms as shown in Figure 9, All experiments have been performed on Intel Core $17-4790 \mathrm{~K} 4.00 \mathrm{GHz}$ machine with $8 \mathrm{~GB}$ main memory. Internal parameters of the GA framework are set as shown in Table I $\mu$ and $\lambda$ denote the number of parents and offspring.

Table I. Configuration of the GA framework

\begin{tabular}{|l|l|}
\hline Population size & 100 \\
\hline$\mu$ and $\lambda$ & 100 \\
\hline Probabilities of crossover/mutation & 0.9 \\
\hline Maximum generations & 30000 \\
\hline
\end{tabular}

\subsection{MMDF Scheduling Technique}

We compare the proposed technique with two different heuristics. The first heuristic schedules SDF graphs independently and performs the processor renaming heuristic. We denote this technique as Base. The Base technique is an iterative algorithm. First, for each mode, it constructs pareto-optimal solutions which are optimized with throughput and the number of processors using a genetic algorithm. Then it selects a initial schedule which satisfies the throughput constraint with the minimum number of processors for each mode. Based on the mapping/scheduling results, it performs the processor renaming heuristic and adjusts the throughput requirement as discussed in the previous section, considering the mode transition delay incurred by the initial schedules. If a schedule does not satisfy the calculated throughput requirement, it is replaced with another schedule which uses one more processor. Unless all scheduling results satisfy the new adjusted throughput requirement in all modes, it repeats the mapping/scheduling with the new adjusted throughput requirement until the mapping/scheduling results satisfy the adjusted throughput requirement. 


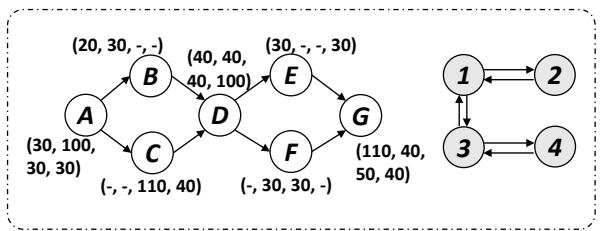

Example 1

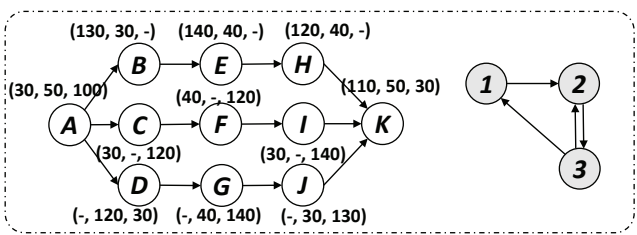

Example 2

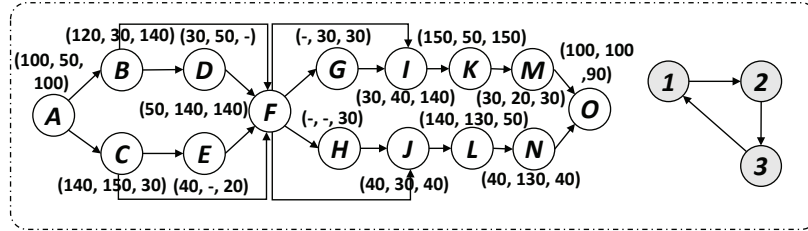

Example 3

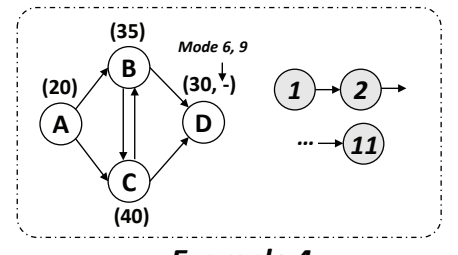

Example 4

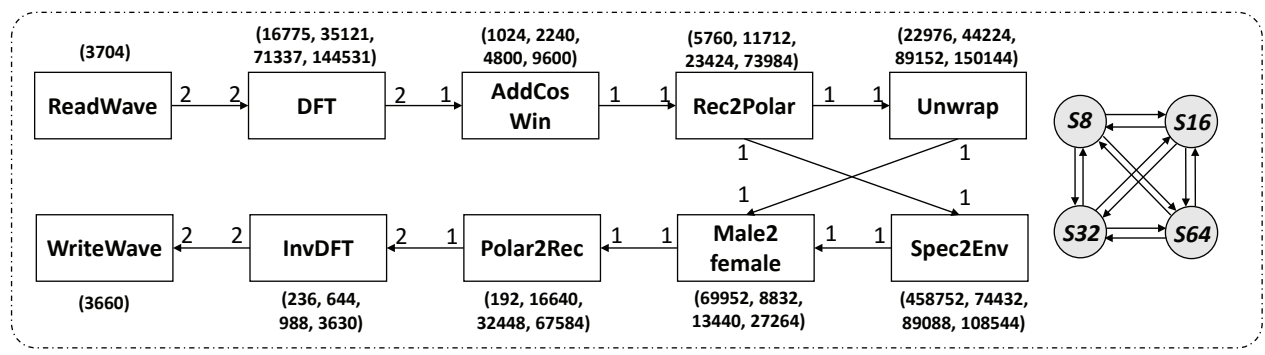

\section{Vocoder}

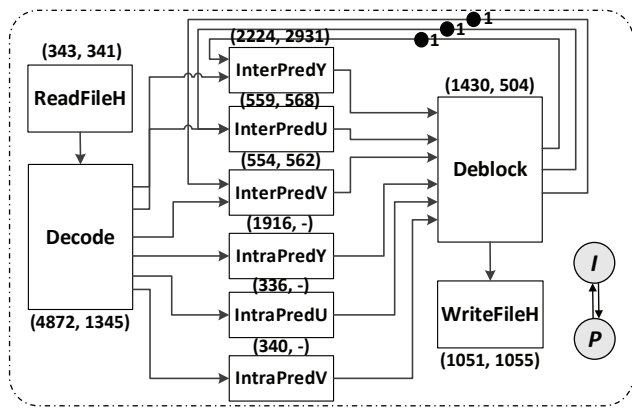

H.264 decoder

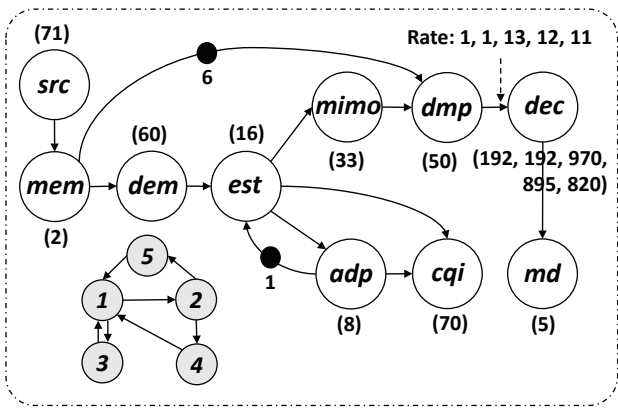

LTE receiver

Fig. 9. MMDF graph examples used in experiments

The second approach fixes task mapping in all modes disallowing task migration as the existing approaches usually assume. This technique is denoted as Fixed. The Fixed technique is implemented in the same GA framework as the proposed framework with disallowing task migration only.

Each MMDF graph consists of a task graph and the associated $M T G$. For all graphs in Figure 9 exclude the vocoder application, Rate ( $p$, mode) for each port $p \in P_{t}$ is one except the input port of node dec in the LTE receiver application. For the vocoder application, port rates are fixed among all modes and specified in the figure. For the task graph of vocoder application in [Zhai 2015], we reduce the number of invocations for specific tasks (from AddCosWin to Polar2Rec) from 128 to 2 by clustering, so the given 
WCETs of those tasks in [Zhai 2015] are multiplied by 64. Also, we allow that each instance of the same node can be mapped onto different processors for data parallelism.

The numbers above or under the tasks in Figure 9 indicate the $W C E T\left(t_{m}\right)$ in each mode. In case that the $W C E T\left(t_{m}\right)$ of a task is constant in all modes, a single number is denoted. For synthetic examples, the WCET of each task is set to an arbitrary value, and the WCET of each task in the H.264 decoder application is set to profiled data with us unit. Also, the WCET of each task in the vocoder and LTE receiver applications is set to a value given in [Zhai 2015] and [Siyoum et al. 2011].

Table II. Configurations for experiments

\begin{tabular}{|c|c|c|}
\hline & $M R C(m)$ & ThrConst \\
\hline Example 1 & $\forall m \in$ Mode, $M R C(m)=5$ & 1/150 iteration/time-unit \\
\hline Example 2 & $\forall m \in$ Mode, $M R C(m)=5$ & $1 / 260$ iteration/time-unit \\
\hline Example 3 & $\forall m \in$ Mode, $M R C(m)=5$ & 1/330 iteration/time-unit \\
\hline Example 4 & $\forall m \in$ Mode, $M R C(m)=5$ & 1/80 iteration/time-unit \\
\hline Vocoder & $\forall m \in$ Mode, $M R C(m)=5$ & 1/500000 iteration/cycle \\
\hline$H .264$ decoder & $M R C(I)=1, M R C(P)=5$ & $1 / 12500$ iteration/us (80 fps) \\
\hline LTE receiver & $\forall m \in$ Mode, $M R C(m)=5$ & 1/1800 sub-frames/time-unit \\
\hline
\end{tabular}

For all configurations in Table II, we compare the following three techniques: Base, Fixed, and Proposed. We assume that the minimum repetition count $(M R C)$ for all modes in each example is set to 5 except the H.264 decoder application, since the mode transition pattern of the H.264 decoder is known and fixed (eg. I-P-P-P-P-P-I-P-P-P-...). Throughput constraints are set arbitrarily with considering the WCET of tasks.

Figure 10 shows the experimental results for all applications. The y-axis indicates the number of required processors and the $\mathrm{x}$-axis presents migration cost $(M C(t))$. To show the viability of the proposed framework, we examine how the mode transition delay takes effect on the number of processors. For each example, we vary the task migration cost or $M C(t)$ using four different values. In the synthetic examples and the vocoder/LTE receiver applications, the task migration cost is fixed as $M C(t)$ for all tasks. In H.264 decoder applications, however, $M C(t)$ is scaled based on the actual task code size for all $t \in T$ : the task migration cost of a task is computed as the product of $M C(t)$ values in $\mathrm{x}$-axis and its task code size.

The results show that the Proposed method requires no more processors than Base and Fixed approaches. The Fixed approach requires more processors in most cases than Base and Proposed approaches. Since the Fixed approach does not allow task migration, the number of required processors to meet the given throughput constraint is independent of the task migration cost. Since the mode transition delay is determined by not only the task migration delay but also the latency in the blocking scheme of task migration, when $M C(t)$ is small, the mode transition delay is mostly dependent on the latency. Therefore, the Base approach shows the similar results to the Proposed approach for small migration costs. However, as $M C(t)$ increases, the Base approach requires more processors than the Proposed approach or could not find a feasible solution for large $M C(t)$ values in cases which are highlighted with a green box in Figure 10.

In the H.264 decoder application, there exists a dominant mode in which all tasks in an MMDF graph are executed. Since the dominant mode creates a critical path in all modes, if a mapping and scheduling result satisfies the throughput constraint in the dominant mode then results in the other modes automatically satisfy the throughput 


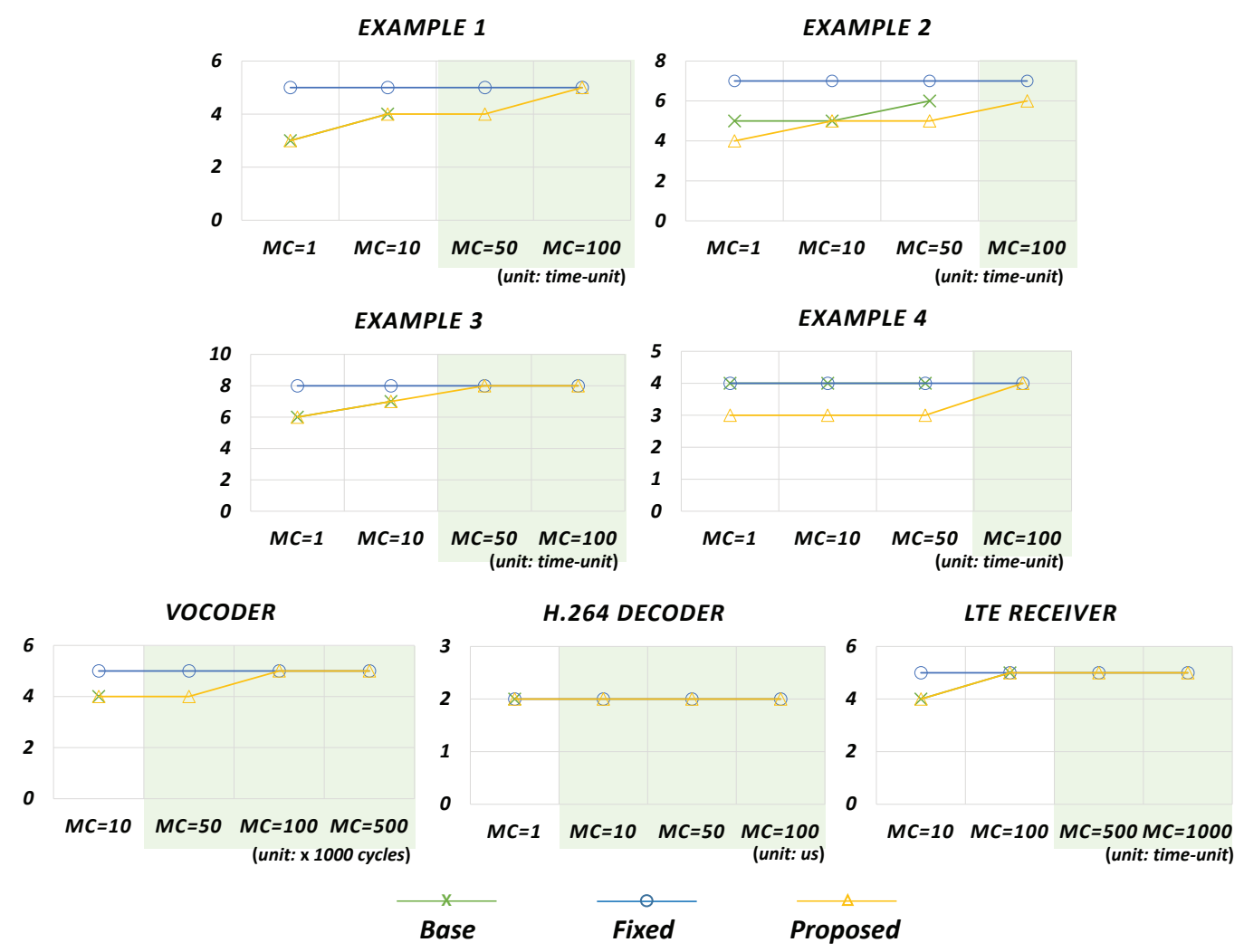

Fig. 10. Comparison results in terms of the number of processors: Base, Fixed and Proposed

constraint. Hence, no task migration is required and Fixed and Proposed approaches produce the same results for the application.

Table III. Experimental results of Proposed in case of $M C(t)=10$

\begin{tabular}{|c|c|c|c|c|}
\hline & $\begin{array}{c}\max _{m \in \text { Mode }} \\
\text { MaxTransDelay }(m)\end{array}$ & $\begin{array}{c}\min _{m \in \text { Mode }} \\
\text { ThrRequire }(m)\end{array}$ & $\begin{array}{c}\text { Output } \\
\text { buffer size }\end{array}$ & MigCost total \\
\hline Example 1 & 150 time-unit & $1 / 120$ & 2 & 20 time-unit \\
\hline Example 2 & 380 time-unit & $1 / 184$ & 3 & 20 time-unit \\
\hline Example 3 & 640 time-unit & $1 / 202$ & 3 & 60 time-unit \\
\hline Example 4 & 70 time-unit & $1 / 66$ & 2 & 50 time-unit \\
\hline Vocoder & 280373 cycles & $1 / 443925$ & 2 & 150000 cycles \\
\hline H.264 decoder & 746 us & $1 / 12350.8$ & 1 & 0 time-unit \\
\hline LTE receiver & 980 time-unit & $1 / 1604$ & 2 & 30 time-unit \\
\hline
\end{tabular}

Table III presents the detailed experimental results from the Proposed approach in Figure 10 when $M C(t)=10$ for instance. The table informs that the throughput which an application should satisfy becomes tighter than the given throughput constraint in Table II due to the mode transition delay. The table also presents the total task migration cost and the required output buffer sizes for benchmark applications. 


\subsection{Scalability of the Proposed Framework}

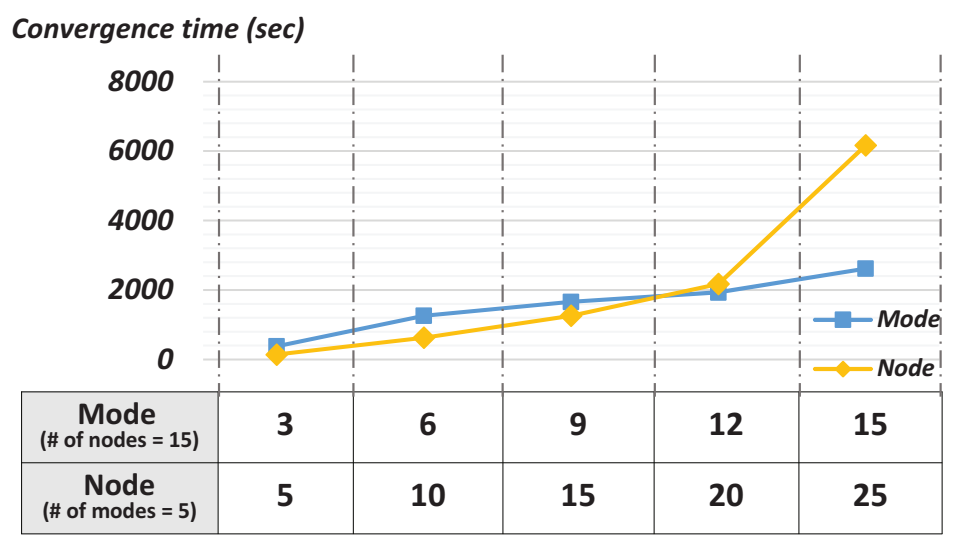

Fig. 11. Experimental result for the scalability property

Because the proposed framework is based on the multi-objective genetic algorithm, its convergence speed depends on the size of solution space. As shown in Figure 7, the size of the solution space depends on the number of nodes and modes. So, we perform experiments for different configurations of these factors. Figure 11] shows the experimental results of the scalability of the proposed framework for synthetic examples. The results show that the number of nodes more contributes to the convergence speed than the number of modes.

\section{CONCLUSION}

In this paper, we address the multiprocessor scheduling problem of a multi-mode dataflow (MMDF) graph allowing task migration with non-negligible mode transition delay. An MMDF graph has a finite set of modes and each mode is specified by an SDF graph. We observe that the mode transition delay should be considered in many streaming applications in which the mode transition occurs frequently, in order to satisfy the throughput constraint. Thus we propose a mapping/scheduling framework based on a genetic algorithm which schedules all SDF graphs simultaneously to minimize the number of processors while keeping the throughput constraint. Also, we propose the formulations to compute the required buffer size and the required throughput performance of the MMDF graph to satisfy the given throughput constraint of the system, by estimating the mode transition delay conservatively from an obtained scheduling result under the assumption of blocking scheme of task migration. To show the viability of the proposed technique, we compare the proposed technique with two other approaches with some synthetic examples and three real applications. Experimental results confirm the superiority of the proposed technique over other approaches.

Because the mode transition delay is conservatively calculated with blocking scheme of task migration, as a future work, we plan to calculate the throughput requirement with more exact model of mode transition.

\section{ACKNOWLEDGMENTS}

This research was supported by a grant to Bio-Mimetic Robot Research Center Funded by Defense Acquisition Program Administration, and by Agency for Defense Development (UD130070ID), Basic Science Research Program through the National Research Foundation of Korea (NRF) funded by the Ministry of 
Science, ICT \& Future Planning (NRF-2013R1A2A2A01067907, 2013R1A1A1013384), and IT R\&D program MKE/KEIT (No. 10041608, Embedded system Software for New-memory based Smart Device).

\section{REFERENCES}

Vagelis Bebelis, Pascal Fradet, Alain Girault, and Bruno Lavigueur. 2013. BPDF: A Statically Analyzable DataFlow Model with Integer and Boolean Parameters. In Proceedings of the Eleventh ACM International Conference on Embedded Software (EMSOFT '13). IEEE Press, Piscataway, NJ, USA, Article 3, 10 pages. http://dl.acm.org/citation.cfm?id=2555754.2555757

Bishnupriya Bhattacharya and Shuvra S. Bhattacharyya. 2001. Parameterized dataflow modeling for DSP systems. Signal Processing, IEEE Transactions on 49, 10 (Oct 2001), 2408-2421. DOI : http://dx.doi.org/10.1109/78.950795

Greet Bilsen, Marc Engels, Rudy Lauwereins, and J.A. Peperstraete. 1995. Cyclo-static data flow. In Acoustics, Speech, and Signal Processing, 1995. ICASSP-95., 1995 International Conference on, Vol. 5. 32553258 vol.5. DOI : http://dx.doi.org/10.1109/ICASSP.1995.479579

Morteza Damavandpeyma, Sander Stuijk, Twan Basten, Marc Geilen, and Henk Corporaal. 2013. Throughput-constrained DVFS for scenario-aware dataflow graphs. In Real-Time and Embedded Technology and Applications Symposium (RTAS), 2013 IEEE 19th. 175-184. DOI :http://dx.doi.org/10.1109/RTAS.2013.6531090

Marc Geilen and Sander Stuijk. 2010. Worst-case Performance Analysis of Synchronous Dataflow Scenarios. In Proceedings of the Eighth IEEE/ACM/IFIP International Conference on Hardware/Software Codesign and System Synthesis (CODES/ISSS '10). ACM, New York, NY, USA, 125134. DOI: http://dx.doi.org/10.1145/1878961.1878985

Marc Geilen, Sander Stuijk, and Twan Basten. 2012. Predictable dynamic embedded data processing. In Embedded Computer Systems (SAMOS), 2012 International Conference on. 320-327. DOI :http://dx.doi.org/10.1109/SAMOS.2012.6404194

Alain Girault, Bilung Lee, and Edward A. Lee. 1999. Hierarchical finite state machines with multiple concurrency models. Computer-Aided Design of Integrated Circuits and Systems, IEEE Transactions on 18, 6 (Jun 1999), 742-760. DOI : http://dx.doi.org/10.1109/43.766725

Hanwoong Jung, Chanhee Lee, Shin haeng Kang, Sungchan Kim, Hyunok Oh, and Soonhoi Ha. 2014. Dynamic Behavior Specification and Dynamic Mapping for Real-Time Embedded Systems: HOPES Approach. ACM Trans. Embed. Comput. Syst. 13, 4s, Article 135 (April 2014), 26 pages. DOI : http://dx.doi.org/10.1145/2584658

Chanhee Lee, Sungchan Kim, Hyunok Oh, and Soonhoi Ha. 2013. Failure-Aware Task Scheduling of Synchronous Data Flow Graphs Under Real-Time Constraints. Journal of Signal Processing Systems 73, 2 (2013), 201-212. DOI:http://dx.doi.org/10.1007/s11265-013-0753-3

Edward A. Lee and David G. Messerschmitt. 1987. Synchronous data flow. Proc. IEEE 75, 9 (Sept 1987), 1235-1245. DOI : http://dx.doi.org/10.1109/PROC.1987.13876

Orlando Moreira. 2012. Temporal analysis and scheduling of hard real-time radios running on a multiprocessor. ser. PHD Thesis, Technische Universiteit Eindhoven (2012).

Firew Siyoum, Marc Geilen, Orlando Moreira, Rick Nas, and Henk Corporaal. 2011. Analyzing synchronous dataflow scenarios for dynamic software-defined radio applications. In System on Chip (SoC), 2011 International Symposium on. 14-21. DOI: http://dx.doi.org/10.1109/ISSOC.2011.6089222

Sander Stuijk, Marc Geilen, and Twan Basten. 2010. A Predictable Multiprocessor Design Flow for Streaming Applications with Dynamic Behaviour. In Digital System Design: Architectures, Methods and Tools (DSD), 2010 13th Euromicro Conference on. 548-555. DOI :http://dx.doi.org/10.1109/DSD.2010.31

Sander Stuijk, Marc Geilen, Bart D. Theelen, and Twan Basten. 2011. Scenario-aware dataflow: Modeling, analysis and implementation of dynamic applications. In Embedded Computer Systems (SAMOS), 2011 International Conference on. 404-411. DOI:http://dx.doi.org/10.1109/SAMOS.2011.6045491

Sander Stuijk, Amirhossein Ghamarian, Bart D. Theelen, Marc Geilen, and Twan Basten. 2008. FSM-based $S A D F$. Technical Report. Citeseer.

Lothar Thiele, Samarjit Chakrabort, and Martin Naedele. 2000. Real-time calculus for scheduling hard real-time systems. In Circuits and Systems, 2000. Proceedings. ISCAS 2000 Geneva. The 2000 IEEE International Symposium on, Vol. 4. 101-104 vol.4. DOI:http://dx.doi.org/10.1109/ISCAS.2000.858698

Maarten H. Wiggers, Marco J. G. Bekooij, and Gerard J. M. Smit. 2008. Buffer Capacity Computation for Throughput Constrained Streaming Applications with Data-Dependent Inter-Task Communication. In Real-Time and Embedded Technology and Applications Symposium, 2008. RTAS '08. IEEE. 183-194. DOI :http://dx.doi.org/10.1109/RTAS.2008.10 
Multiprocessor Scheduling of a Multi-mode Dataflow Graph Considering Mode Transition Delay39:21

Jiali Teddy Zhai. 2015. Adaptive streaming applications : analysis and implementation models. ser. PHD Thesis, Universiteit Leiden (2015). 